\title{
Thatcher's children: Development and the Thatcher illusion
}

\author{
Michael B Lewis \\ School of Psychology, Cardiff University, PO Box 901, Cardiff CF10 3YG, Wales, UK; \\ e-mail: LewisMB@cardiff.ac.uk \\ Received 11 April 2003, in revised form 13 October 2003
}

\begin{abstract}
Children do not show the same recognition disadvantage for inverted faces as adults do. It has been suggested that this is because the configural encoding (which is disrupted by inversion) becomes more useful or available as we get older. The distinction between configural processing and featural processing, however, is not always clear-it may be a dichotomy or a continuum. The perceived normal-to-grotesque switch in the Thatcher illusion was investigated, as the image was rotated, by people aged between 6 and 75 years. No effect of age was found, with young children showing the same effects as adults-the switch occurring at about $72^{\circ}$. The development of face processing and the nature of facial configural encoding are discussed in the light of this result.
\end{abstract}

\section{Introduction}

A well-established finding in face recognition is that inverted faces are harder to recognise than upright faces and this effect is larger than for other forms of stimuli (eg Yin 1969). One explanation for this finding is that faces (and possibly other images for which we have expertise) are recognised holistically. Inversion disrupts this holistic or configural processing, which implies that we need to make use of more local or feature-based strategies to recognise inverted faces (Tanaka and Farah 1993).

Carey and Diamond (1977) demonstrated that children under the age of 8 years do not show this disproportional decrement caused by inversion of faces. This result suggested that younger children process faces according to their parts and then develop a more configural or holistic strategy as they get older (possibly by the age of 10 years). That is, there is a shift in the method of processing faces. This hypothesis has been disputed by a variety of researchers (eg Flin 1985; Tanaka et al 1998; Valentine 1988). An alternative explanation of Carey and Diamond's data [offered by Tanaka et al (1998)] is that 6-year-olds are poorer than older children at recognising upright faces. As the children get older they get better at recognising upright faces but remain stable at inverted faces. There is, therefore, no shift in the way faces are processed but a gradual increase in the amount of configural encoding.

An experiment has been designed to explore this change in configural encoding as children get older. It makes use of a well-known illusion in the face-processing literature-namely the Thatcher illusion (Thompson 1980). This illusion is generated by inverting the eyes and mouth of a face. The resulting image looks grotesque until the whole thing is inverted when it looks almost normal. A widely accepted account for this illusion is that the Thatcherisation processes change only the configuration of features and not the features themselves. Inversion of the whole image disrupts the configuration and so the configural changes are no longer apparent (see Bartlett and Searcy 1993). This illusion has been used, therefore, to investigate whether configural encoding is disrupted by particular transformations (eg Lewis and Johnston 1997). Also, it has been used to investigate how configural encoding switches to featural encoding as the orientation of faces changes from upright to inverted (Lewis 2001; Sjoberg and Windes 1992; Stürzel and Spillmann 2000). 
Stürzel and Spillmann (2000) rotated Thatcherised images until they reportedly switched between being normal and grotesque looking. Using this procedure, they found that a normal-looking inverted Thatcherised face began to look grotesque after a rotation of about $97^{\circ}$ towards being upright. They therefore suggested that the switch between featural and configural processing took place somewhere close to this region. However, I suggested that the methodology employed would lead to an overestimation of between $15^{\circ}$ and $30^{\circ}$ (Lewis 2001). This was because the images were rotated at a speed of $30^{\circ} \mathrm{s}^{-1}$ by the experimenter and the time it would take for the participant to voice a stop action, and the experimenter to act upon this, would lead to about 0.5 to $1 \mathrm{~s}$ delay. I further disputed whether such a sudden switch in processing styles took place, suggesting rather that there was a gradual change. In that paper, reaction times to singly presented faces were recorded for a Thatcherised or non-Thatcherised decision. A gradual change through rotation was found to occur suggesting that encoding of faces might vary on a continuum from featural to configural and that differing degrees of rotation disrupt encoding differentially along this continuum. Murray et al (2000) also found a gradual change in the perception of the Thatcher illusion at different rotations, but their task required a bizarreness rating.

The research to date shows two differing effects. I (Lewis 2001) and Murray et al (2000) showed a gradual change in reaction times or ratings through rotation, whereas Stürzel and Spillmann (2000) showed an abrupt change. These two findings can be reconciled if we appeal to the research on categorical perception. Gradually changing the wavelength of light can process a colour-naming category shift, whereas a typical exemplar of a particular colour (ie the focal colour) is named faster than a hue that only just falls within that colour category (for an overview, see Harnad 1987). Importantly for the current study, Raskin et al (1983) described how categories of colours become fixed at an early age of development. This suggests that even stimuli that show a gradual perceptual change allow participants to report an abrupt category shift. Arguably, this is what the participants in Stürzel and Spillmann's experiment were doing, whereas my participants were responding to the gradual change to the perceptual properties of the image. At some point through rotation, the grotesque nature of the face becomes apparent (giving the abrupt shift) but it can still vary in how easy it is to detect (giving the gradual change observed in reaction-time measures). I am here concerned with the angle of rotation at which configural encoding and hence the grotesque nature of the face can be found for participants of different ages. Reaction-time changes would be difficult to interpret across participants whose ages ranged between 6 and 75 years, and so the Stürzel and Spillmann style experiment was employed here.

The developmental change in face processing, if indeed one takes place, could be interpreted as a change in the relative importance of configural and featural encoding. Younger children may employ both methods equally but configural processing may develop as the child gets older. This account would be consistent with Tanaka et al's (1998) findings. Such an hypothesis would make direct predictions concerning the Thatcher illusion. If, as I have speculated, there is a continuum of encoding and the weighting of this encoding gradually changes through development (Lewis 2001), children should see any switch in the Thatcher illusions (from normal to grotesque) appear after a larger degree of rotation than is necessary for adults, the reason being that children have less total configural encoding available to them and so rotation will have to be greater to allow sufficient encoding to be available to detect the grotesque nature.

If, indeed, the nature of face encoding is dichotomously split into featural and configural, then it is possible that the degree of rotation at which all configural encoding becomes available for children is the same as where it becomes available for adults. In this situation, it would be expected that age should not affect the degree at which the switch is perceived to take place. I test here these hypotheses by allowing participants 
to identify at what point the normal-to-grotesque switch is perceived for a Thatcherised image by people of different ages. The procedure was similar to that employed by Stürzel and Spillmann (2000), although only one direction of rotation was employed.

\section{Method}

\subsection{Participants}

Participants were sixty-six members of the public who visited Techniquest (Cardiff Bay, UK) one day during National Science Week. They had a mean age of 23.1 years $(\mathrm{SD}=18.8$ years), but individual ages ranged from 6 to 75 years. None of the subjects had previously encountered the Thatcher illusion. All participants were tested individually.

\subsection{Stimulus}

A colour front-view image of Gareth Gates was used to generate the stimulus. Gareth Gates is a British pop star who has received considerable television exposure including playing a significant role in a popular British Telethon the night before the experiment. The image was Thatcherised by having the eyes and mouth of the image inverted: oval masks were used to select the features and 'feathering' (a tool available in Corel Photopaint ${ }^{(i x)}$ ) of the mask was used to avoid any sharp contours. The Thatcherised image was blown up and printed onto a circular sheet with a diameter of 12 inches. The sheet was attached to a larger circle so that it could rotate freely. The top of the head contained a pointer that pointed to different numbers on the larger circle as

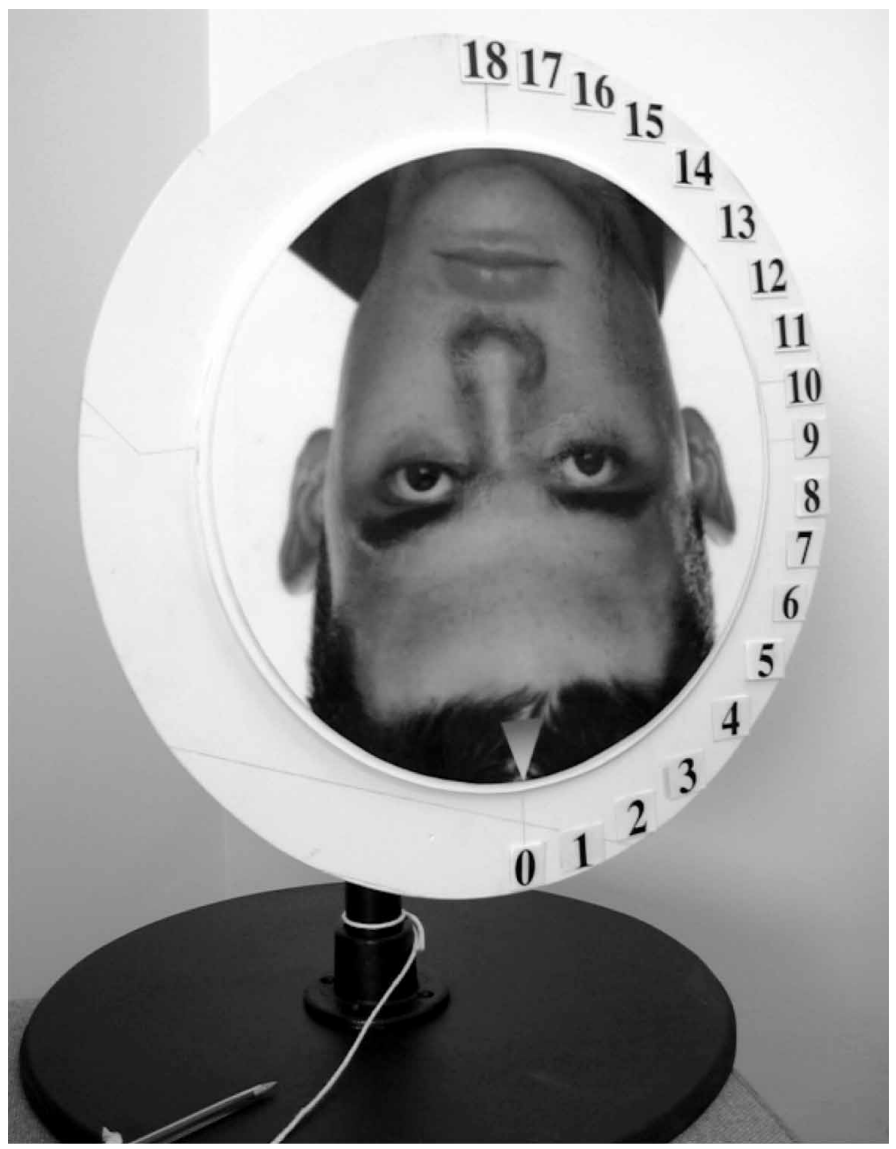

Figure 1. The setup employed in the experiment. The inner disc containing the face was freely rotated whilst the outer disc remained fixed so that the pointer indicated the degree of rotation at which the grotesque appearance became apparent. 
the image was rotated. The numbers were fixed to the outside of the larger circle and went from ' 0 ' at the bottom to ' 18 ' at the top, over the right-hand circumference in steps of $10^{\circ}$ (see figure 1). The fixed and rotating discs were mounted vertically on a stand that stood on a table.

\subsection{Procedure}

Volunteers were asked to sit on a chair with the rotating disc at approximately eye level straight in front of them. The Thatcherised face always started in the inverted position (pointer at 0). The participants were asked whether the face looked okay. Many said "yes", but some said "no, he is upside down". The image was then rotated slowly until it was upright (pointer on 18). The participant was asked whether the face still looked okay. The types of responses were that it looked "weird", "ugly", "strange", "funny", "horrible", etc (it is worth noting that none of the participants used the term "grotesque"). The experimenter then used the participant's own term to suggest that at some point the image moves from being normal looking to being weird, strange, horrible, or whatever their response was, and that we would like to know at what point the image starts to look like that. The participants were then encouraged to freely rotate the disc until they thought that the image just became whatever adjective they had chosen. Some of the younger children needed help with the experimenter turning the disc for them. The number on which the pointer was when the change was perceived to occur was recorded. If the pointer was between numbers, then a half measure was recorded giving a resolution of $5^{\circ}$ angle of rotation. The participant's age was recorded after they had completed the task.

\section{Results}

All participants reported seeing a change occur somewhere between $35^{\circ}$ and $130^{\circ}$ $\left(0^{\circ}\right.$ always means that the face was inverted $)$. The mean change was reported at $72.0^{\circ}$ $\left(\mathrm{SD}=22.5^{\circ}\right)$. All participants were able to choose a degree where the change took place and none of them reported that they could not do the task because the change was too gradual.

A regression analysis was conducted to investigate whether the point of change varied for participants of different ages. The age of participants was skewed and so it was $\log$ transformed. This also meant that any effects that were presented at younger ages would contribute more. Figure 2 shows the pattern of data. There was a slight increase in the angle of rotation of the perceived change for older participants, but this effect was not significant $\left(t_{64}=1.273, p>0.05\right.$ - this trend was in the opposite direction to that predicted by a processing shift). The linear effect of age was also not significant. In order to check that there was not any kind of step-function change with age, a series of unpaired $t$-test comparisons were made between (i) participants under 10 years of age and those over 10 years $\left(t_{64}=0.218\right)$; participants under 8 years of age and those over 12 years $\left(t_{50}=0.525\right)$; and participants under 10 years of age and those between 20 and 40 years $\left(t_{41}=0.655\right)$. In response to an observation from a reviewer, a comparison between those aged under 25 years and those aged over 25 years was performed. This analysis did reveal a significant effect $\left(t_{64}=2.418\right.$, $p<0.05)$. There was no a priori reason for selecting to partition the participants in this way and so such a result must be treated with caution because it suggests an increased possibility of accepting an hypothesis when it is not true (ie Type 1 error).

As neither the regressions nor the hypothesis-related $t$-tests were significant, it is possible that participants were not able to do the task and just responded randomly. The fact that the data are not purely random can be shown by the difference of the responses to the predicted average if responses had been random (ie $90^{\circ}$ ). For participants over 10 -years-old, the $95 \%$ confidence interval of mean performance was between $63.3^{\circ}$ 


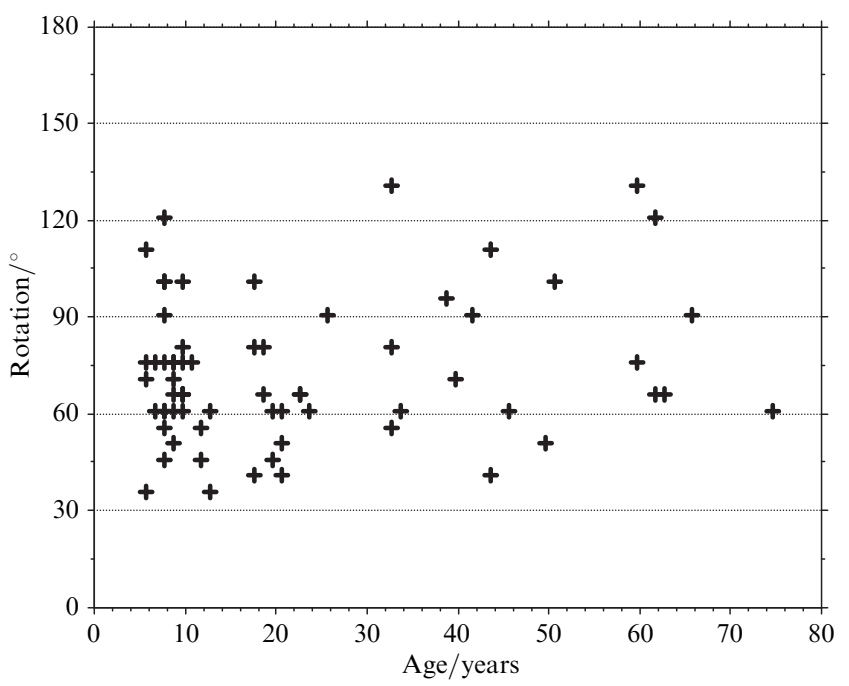

Figure 2. The degree of rotation of the perceived switch from normal to 'grotesque' as predicted (or rather not predicted) by age of the participant.

and $79.5^{\circ}$ rotation. This does not include $90^{\circ}$ (the hypothesised average if performance had been random, $t_{37}=4.643, p<0.001$ ). Similarly for children aged 10 years and less, the $95 \%$ confidence interval ranges from $65.1^{\circ}$ to $80.2^{\circ}$, meaning that the hypothesis that average performance was $90^{\circ}$ can again be rejected $\left(t_{27}=4.687, p<0.001\right)$. Both groups, therefore, show that the average response was significantly less than $90^{\circ}$ of rotation and so performance was not random.

It can be observed that there was a close similarity between the degrees of rotation chosen by those children aged 10 years and less and children over 10 years of age. While no statistical test can show that the two groups were identical, it is possible to use the data collected to place limits on how dissimilar the two groups were. Again, using confidence intervals, we can say with $95 \%$ certainty that the difference between the degree of rotation chosen by children aged 10 years and less and those over 10 years of age was less than $10^{\circ}$ (the estimated difference is, in fact, just $1.5^{\circ}-72.5^{\circ}$ for children 10 years old and less versus $71.0^{\circ}$ for children over 10 years of age).

\section{Discussion}

The first result that was apparent from conducting the experiment was that none of the participants found it difficult to select a point at which the image switched. While the degree of rotation varied a great deal, no participant stated that it was just too gradual to make a decision. This may suggest that the dichotomy of encoding is correct or it may suggest that participants were able to follow the instructions because of a categorical shift brought on by a gradually perceived change. In this second case, the variation seen between subjects could represent differences in where their category boundaries lie.

The second result to note is that there is no real significant effect of age on degree of rotation for the change to occur around the age that would be predicted by Carey and Diamond's (1977) processing shift. The presence of an effect was explored in a number of ways and none of these showed anything even approaching a significant effect of age, with younger participants requiring greater rotation to see the grotesque nature of the face. One significant finding was that those over 25 years of age required greater rotation than those under 25 years of age. This effect was in the opposite direction to that predicted by an increase in configural encoding through development. 
While it is possible that this is a real effect, it is also possible that it is a spurious result produced by a posteriori analysis of the data.

The subsequent analysis, however, does allow us to make some inferences from the data. The average rotation for the switch chosen by those over 10 years old is significantly less than $90^{\circ}$. This indicates that their performance is not random. Similar analysis showed that children aged 10 years and less also gave an average response under $90^{\circ}$ and so they too were not performing randomly. The most convincing evidence that there is not any effect of age on the degree of rotation of the switch comes from the analysis of the confidence interval. From this it can be stated that, if we assume that there is a change that takes place between those aged 10 years and less and those aged over 10 years, then the size of this change must be less than $10^{\circ}$ of rotation. If there is a processing shift, then its influence is so small as to be almost negligible.

We can be reasonably confident from the results presented here that children as young as 6-years-old see the Thatcher illusion as adults do. All the children showed visible surprise as the face was rotated to the upright position. Indeed, one 6-year-old girl took quite a while to do the experiment because she kept laughing whenever the image was shown in the upright orientation. It can also be seen that there is little, and possibly even no, effect of age on the degree of rotation at which the switch between normal and grotesque takes place. I failed to find any differences in how children and adults perceive the Thatcher illusion.

This result has important consequences for theories of encoding and development. In my earlier paper (Lewis 2001) I hypothesised that encoding varied on a continuum from featural to configural. Further, Tanaka et al (1998) showed that during development recognition of whole faces becomes better, whereas recognition of inverted faces remains stable. This suggests that improvement due to development is in configural encoding alone. Maybe it is the case that configural encoding becomes more robust to rotation as one gets older. If this were the case then one would expect to see greater rotation required for the younger participants to see the grotesque nature of the face. The prediction suggests more than just the $10^{\circ}$ maximum difference allowed for by the current results. The current data, therefore, require us to reject this model of faceencoding development. Instead, it must be the case that whatever configural encoding is required to see the Thatcher illusion, it is present from an early age and the degree of rotation at which it is available is unchanged by development.

If continuum of configural encoding suggested by me earlier (Lewis 2001) is correct, then the data from the current experiment can be reconciled with previous research in the following way. It may be the case that, encoding that is robust to large amounts of rotation (ie more featural in nature) is developed before encoding that is disrupted by small amounts of rotation. It is this earlier configural encoding that seems necessary for the Thatcherised face to appear grotesque. Therefore, the degree of configural encoding required to see the grotesque Thatcherised face is developed at an age lower than 6 years, but other configural encoding (that which is even less robust to small amounts of rotation) continues to develop as one gets older.

The result from the current experiment, however, can also be reconciled with dichotomous configural and featural encoding. The age at which configural encoding becomes available must be lower than 6 years and its nature (in terms of sensitivity to rotation) does not change as one gets older. What may change, however, is the efficiency and effectiveness with which this configural encoding is employed in general face recognition. One result that is difficult to explain with this dichotomy of encoding is why Stürzel and Spillmann (2000) found the grotesque appearance of the Thatcherised face appearing at $97^{\circ}$ rotation whereas here (albeit with different stimulus) an angle of $72^{\circ}$ was found. If there were a dichotomous split, then we would expect different tasks to find the abrupt changes to take place at the same degree of rotation. 
What has been learnt from this experiment is that children perceive the Thatcher illusion in much the same way as adults do. Some level of configural encoding of faces, therefore, appears to become available to children and adults at the same degree of rotation of a face. Whether even more configural encoding is available to adults and not children, at only small degrees of rotation from upright, remains to be seen.

Acknowledgments. This research was made possible through EPSRC grant GR37777 and the EPSRC 'Meet the scientists' initiative. I would like to thank the staff of Techniquest science centre in Cardiff, particularly Louise Eccleston and Keith Powney, and also copresenter Ilyas Khan for conducting much of the experiment.

\section{References}

Bartlett J C, Searcy J, 1993 "Inversion and configuration of faces" Cognitive Psychology 25281 - 316

Carey S, Diamond R, 1977 "From piecemeal to configurational representation of faces" Science $195312-314$

Flin R H, 1985 "Development of face recognition: An encoding switch?" British Journal of Psychology $76123-134$

Harnad S, 1987 "Psychophysical and cognitive aspects of categorical perception: A critical overview", in Categorical Perception: The Groundwork of Cognition Ed. S Harnad (Cambridge: Cambridge University Press) pp 535-565

Lewis M B, 2001 "The Lady's not for turning: Rotation of the Thatcher illusion" Perception 30 $769-774$

Lewis M B, Johnston R A, 1997 "The Thatcher illusion as a test of configural disruption" Perception $26225-227$

Murray J E, Yong E, Rhodes G, 2000 "Revisiting the perception of upside-down faces" Psychological Science $11492-496$

Raskin L A, Maitial S, Bornstein M H, 1983 "Perceptual categorisating of color: A life-span study" Psychological Research $45135-145$

Sjoberg W, Windes J D, 1992 "Recognition times for rotated normal and Thatcher faces" Perceptual and Motor Skills $751176-1178$

Stürzel F, Spillmann L, 2000 "Thatcher illusion: Dependence on angle of rotation" Perception 29 $937-942$

Tanaka J W, Farah M J, 1993 "Parts and wholes in face recognition" Quarterly Journal of Experimental Psychology A $\mathbf{4 6} 225$-246

Tanaka J W, Kay J B, Grinnell E, Stansfield B, Szechter L, 1998 "Face recognition in young children: When the whole is greater than the sum of the parts" Visual Cognition 5 479-496

Thompson P, 1980 "Margaret Thatcher: a new illusion" Perception 9 483-484

Valentine T, 1988 "Upside-down faces: A review of the effect of inversion upon face recognition" Memory \& Cognition $79471-491$

Yin R K, 1969 "Looking at upside-down faces" Journal of Experimental Psychology 81 141-145 


\section{PERCEPTION}

VOLUME 322003

www.perceptionweb.com

Conditions of use. This article may be downloaded from the Perception website for personal research by members of subscribing organisations. Authors are entitled to distribute their own article (in printed form or by e-mail) to up to 50 people. This PDF may not be placed on any website (or other online distribution system) without permission of the publisher. 